\title{
Catalytic Strategies for the Production of Renewable Fuels and Chemicals
}

\author{
Ya-Huei Chin \\ Department of Chemical Engineering and Applied Chemistry, University of Toronto \\ Toronto, Canada \\ Cathy.Chin@utoronto.ca
}

Lignocellulosic biomass is an attractive renewable feedstock for synthesizing next generation fuels and chemicals. There are significant challenges in processing this feedstock, because of its structural complexities and high oxygen contents. I will review the various catalytic strategies in processing this feedstock, describe the challenges in this area, and discuss the recent advancement. Specifically, the chemical technology requires selective removal of oxygen heteroatoms with hydrogen, through catalytic pathway turning. This is achieved by coupling of chemistry and transport events at the various length scales, designing catalytically active sites that promote the cascade reactions, and effectively activating and transporting the hydrogen. I will describe the technology for processing the pyrolysis derived oxygenates to hydrocarbon fuels and chemicals and for converting phenolic compounds derived from lignin to higher value products. Emphasizing within this talk is about the design of catalyst structures and their specific catalytic functions in activating the oxygenates and hydrogen. This fundamental understanding of catalytic chemistry, when couples with reactor design and optimization, allows us to advance the atomic and energy efficiencies of the processes. 